\title{
PARTICIPANTES DE ENSAIOS CLÍNICOS EM ONCOLOGIA: PERFIL E ASPECTOS ENVOLVIDOS NAS SUAS DECISÕES
}

\author{
PARTICIPANTS OF ONCOLOGIC CLINICAL TRIALS: PROFILE \\ AND ASPECTS INVOLVED IN THEIR DECISIONS
}

\author{
PARTICIPANTES DE ENSAYOS CLÍNICOS EN ONCOLOGÍA: PERFIL \\ Y ASPECTOS IMPLICADOS EN SUS DECISIONES
}

\author{
Karla Patrícia Cardoso Amorim ${ }^{1}$ \\ Volnei Garrafa ${ }^{2}$ \\ Alana Dantas de Melo $^{3}$ \\ Andressa Vellasco Brito Costa ${ }^{4}$ \\ Gabriella Caldas Leonardo Oliveira ${ }^{5}$ \\ Heitor Giovanni Lopes ${ }^{6}$ \\ Eduardo Judene da Silva Pereira ${ }^{7}$ \\ Francisco Ademar Fernandes Júnior ${ }^{8}$
}

Resumo Estudo sobre os participantes de ensaios clínicos na área de oncologia, discutindo o paradoxo entre progresso científico e iniquidade social. Buscouse conhecer quem são essas pessoas e analisar aspectos envolvidos nas suas decisões, com base em entrevistas e documentos. Houve maior participação feminina. Os participantes tendem a ter poucos anos de estudo formal e baixa renda. A maioria é aposentada e do lar e não tem assistência à saúde privada. Suas decisões giraram em torno da busca pela cura ou melhora, e pela garantia de acesso regular a cuidados integrais de saúde e medicamentos. A assinatura do termo de consentimento livre e esclarecido não garantiu a expressão da autonomia, pois informações essenciais como os objetivos, riscos e cuidados pós-estudo são praticamente desconhecidas. Os participantes da pesquisa tendem a não compreender os objetivos da investigação, ou superestimam os benefícios médicos diretos de sua participação, sem consciência dos riscos envolvidos e do que significa uma pesquisa. Os resultados deveriam incitar ao exercício e ao diálogo mais críticos entre os diferentes atores e instituições envolvidos na área da pesquisa com seres humanos, objetivando promover uma ciência consciente e responsável, que impeça que pessoas sejam colocadas em situação de desigualdade, vulnerabilidade e sofrimento moral.

Palavras-chave sujeitos de pesquisa; ensaios clínicos; iniquidade social; ética em pesquisa; neoplasias.
Abstract This is a study about the participants of clinical trials in the field of oncology that discusses the paradox between scientific advances and social inequality. We sought to get to know these people and to analyze the aspects involved in their decisions based on interviews and documents. Female participation was more expressive. The participants tend to have low schooling and low income. Most of them are retirees and housewives, and do not get assistance from the private health system. Their decisions revolved around the search for cure or recovery, as well as for the assurance of regular access to comprehensive health care and medicines. Signing the free and informed consent form did not guarantee the expression of autonomy, because crucial information, such as the goals, risks and post-study care are virtually unknown. The research participants tend to not understand the goals of the investigation, or they overestimate the direct medical benefits of their participation, and they tend to be unaware of the risks involved and of what does a research mean. The results should stimulate more critical practices and dialogues among the different players and institutions involved in the field of the research with human subjects, with the goal of promoting a science that is conscious and responsible, and that prevents people from being put in situations of inequality, vulnerability and moral suffering.

Keywords research subjects; clinical trials; social inequality; ethics in research; neoplasias. 


\section{Introdução}

O incentivo à pesquisa oncológica é de grande importância. Segundo o entendimento da International Agency for Research on Cancer (IARC) da Organização Mundial da Saúde (OMS), o câncer é um problema de saúde pública, o que corrobora a pertinência de se abordar tal assunto. Tal perspectiva assume maior relevância entre os países em desenvolvimento, onde se espera que, nas próximas décadas, o impacto do câncer na população corresponda a $80 \%$ dos mais de 20 milhões de novos casos estimados para 2025 (Inca, 2015).

Segundo estimativa mundial realizada em 2012 pelo Projeto Globocan/ IARC, dos 14 milhões de casos novos estimados (excetuando-se o câncer de pele não melanoma), mais de $60 \%$ ocorreram em países em desenvolvimento. Em se tratando de mortalidade a situação se agrava, pois foi constatado que dos 8 milhões de óbitos previstos, 70\% ocorreram nesses mesmos países (Inca, 2015).

Entretanto, é necessária a consciência crítica de que as pesquisas clínicas, especificamente os ensaios clínicos, tornaram-se no final do século passado e início deste, definitivamente, uma atividade industrial (Garrafa e Lorenzo, 2009). Nessa perspectiva, é notório que o poder do mercado farmacêutico passou a sobressair, independentemente de suas ações terem cunho positivo ou negativo no tocante à população.

Inseridos nesse cenário científico-industrial-tecnológico das pesquisas clínicas, encontram-se os pacientes oncológicos/participantes de pesquisa, os quais, em países com grandes desigualdades sociais, podem se tornar especialmente vulneráveis. Nesse contexto, é indispensável questionar-se a respeito da autonomia e da dignidade do ser humano: existe autonomia onde faltam meios para suprir as necessidades mais básicas do ser humano? Com relação ao termo de consentimento livre e esclarecido (TCLE): será que, em estado de vulnerabilidade socioeconômica e analfabetismo, não se teria, na maioria das vezes, no máximo um termo de consentimento meramente informado e assinado por um paciente passivo, ao invés de livre e esclarecido?

Outra demanda necessária e urgente no debate a respeito da saúde pública e ética em pesquisa é por investigação sobre o motivo pelo qual os sujeitos aceitam participar de pesquisas clínicas. Seria, principalmente, a falta de opção de tratamento, a dificuldade para obter medicações e exames complementares de alta complexidade (Zoboli e Fracolli, 2001), diante das dificuldades de acesso ao serviço público de saúde em países como o Brasil? Ou seria um sentimento realmente solidário e consciente, que objetive contribuir com o progresso da ciência? Ou, ainda, a busca da possibilidade de cura?

Uma revisão crítica da literatura realizada por Dainesi e Goldbaum (2014) com a finalidade de examinar a tomada de decisão dos pacientes participantes 
de pesquisas biomédicas revelou alguns possíveis motivos: busca por melhores cuidados de saúde, benefícios pessoais, recompensas financeiras e altruísmo. Além disso, os autores evidenciaram que essas razões poderiam variar de acordo com algumas circunstâncias e características, quais sejam: a doença a ser tratada, a fase de estudo, prognósticos, ambiente socioeconômico e cultural. Nappo, Iafrate e Sanchez (2013) relatam, em estudo similar, que as motivações mais presentes entre as enumeradas pelos participantes de estudos clínicos foram o ganho financeiro e a alternativa terapêutica. O altruísmo, nesse caso, não apareceu com frequência e, quando presente, foi considerado um motivo secundário. No entanto, tais perspectivas contrariam os resultados de Zammar e colaboradores (2010), os quais apontam o altruísmo como o principal motivo para os pacientes brasileiros participarem de ensaios clínicos, sendo o reembolso monetário o fator menos importante.

O fato é que existem poucos trabalhos dessa natureza, seja no âmbito brasileiro ou mesmo no contexto internacional, com a finalidade de avaliar tais questionamentos. Caso sejam pertinentes as interrogações a respeito de aspectos que aumentam a vulnerabilidade dos sujeitos envolvidos nos estudos clínicos, é necessário refletir sobre o problema e atualizar o debate a respeito das pesquisas desse tipo. Na verdade, muito pouco se sabe a respeito desses participantes de pesquisa, pois pouca atenção é dispensada a eles. Quem são? Que informações são transmitidas, antes do início dos ensaios clínicos, a essas pessoas? Quais os cuidados a eles dispensados durante e, principalmente, após o término das pesquisas?

O presente trabalho foi desenvolvido exatamente com o objetivo de compreender melhor tais questões. Buscou-se realizar um estudo com o intuito de conhecer e ouvir os participantes de ensaios clínicos, bem como analisar os aspectos envolvidos na sua participação em estudos dessa natureza, em um centro de pesquisa clínica na área da oncologia da cidade de Natal, estado do Rio Grande do Norte, localizado na Região Nordeste do Brasil. A intenção é discutir, com base em um estudo de caso, o paradoxo entre o progresso científico e um contexto demasiadamente iníquo.

\section{Percurso metodológico}

Trata-se de uma pesquisa observacional, do tipo estudo de caso, de caráter descritivo e analítico, com abordagem qualitativa e quantitativa. A junção das abordagens qualitativa e quantitativa foi empreendida de forma complementar, e não antagônica; em um dado momento, as duas abordagens se imbricaram, ajudando a compor a descrição da realidade.

O trabalho foi desenvolvido em duas etapas. A primeira, de caráter quantitativo, teve como objetivo mostrar o perfil socioeconômico (idade, 
sexo, grau de escolaridade, renda familiar, lugar de residência, acesso a assistência de saúde privada etc.). Foram estudados todos os 62 participantes dos 16 ensaios clínicos desenvolvidos no centro de pesquisa entre dezembro de 2006 e dezembro de 2012, os quais constituíram a população do presente trabalho.

Na segunda etapa, de cunho qualitativo, realizaram-se entrevistas semiestruturadas com 11 sujeitos de pesquisa, os quais estavam participando ou haviam participado dos estudos clínicos. Esses sujeitos foram identificados pela letra S, seguida de um número. Utilizou-se como critério para determinar essa quantidade de participantes a repetição de sentidos nos respectivos discursos. A seleção foi estabelecida por conveniência, ou seja, entrevistaram-se os sujeitos, aos quais se conseguiu ter acesso por meio de convite e mediação realizada pelo centro de pesquisa. É importante ressaltar que nos estudos qualitativos a amostragem não obedece a critérios numéricos, mas à possibilidade de permitir aprofundamento e abrangência na compreensão de uma situação (Minayo, 2000).

Buscou-se captar, por meio dos depoimentos dos entrevistados, os seguintes dados: decisão e motivação para participar de estudos clínicos; experiência de ser voluntário de pesquisa em saúde; processo de consentimento livre e esclarecido; conhecimento a respeito dos riscos e benefícios aos quais foram submetidos; e os cuidados a eles dispensados durante e após o estudo.

Os critérios de inclusão dos sujeitos nessa fase foram: ter participado ou estar participando de um ensaio clínico; ser maior de 18 anos e não apresentar algum tipo de alteração, distúrbio, incapacidade ou doença mental capazes de alterar ou impedir a sua capacidade de entendimento no momento da realização da entrevista.

Para realização dessas entrevistas, utilizou-se um roteiro e um gravador, tendo-se obtido anteriormente o consentimento dos entrevistados. Posteriormente, tais entrevistas foram transcritas na íntegra.

Antes da segunda fase da pesquisa, foram feitas duas entrevistas, para testar a validade do roteiro quanto à clareza e à exatidão em relação aos objetivos propostos. Esse procedimento também permitiu evidenciar a imprecisão e a complexidade de algumas questões, bem como a possibilidade de causarem algum constrangimento. Pequenos ajustes foram, então, realizados.

Para registro e análise dos dados quantitativos, utilizou-se o auxílio do programa SPSS para Windows. Tal processo de análise foi feito com base em frequências absolutas, percentuais, médias e distribuições.

O tratamento dos depoimentos colhidos nas entrevistas se deu conforme os passos explicitados por Bardin (2009) acerca da análise de conteúdo. Foi, ainda, utilizado o software NVIVO como ferramenta auxiliar para a análise das entrevistas, com o propósito de organizar o material e proporcionar a visualização quantitativa (frequência de palavras) do que foi verbalizado. 
Em termos gerais, a análise temática de conteúdo foi desdobrada em três etapas. A primeira, caracterizada como pré-análise, consistiu na organização propriamente dita do material compilado. Essa fase iniciou-se com uma leitura 'flutuante' e de leituras repetidas do material, procurando-se um sentido geral do que estava escrito e identificando-se, posteriormente, os pontos convergentes, representativos e significativos em relação ao tema. Desde a pré-análise foram determinadas operações de recorte do texto em unidades comparáveis de categorização para análise temática e de modalidade de codificação para registro dos dados, que foram efetivadas na etapa seguinte.

A segunda fase da análise de conteúdo foi de exploração do material empírico, consistindo essencialmente em operações de codificação e decomposição. Foram definidas sete categorias de análise, buscando responder às seguintes questões: a) Quem são esses pacientes/participantes dos ensaios clínicos? b) Como os pacientes/participantes ficaram sabendo da pesquisa e por que decidiram participar? c) Como se deu o processo de obtenção do TCLE? d) Os pacientes/participantes conhecem os riscos e benefícios a que estiveram expostos? e) Quais os cuidados dispensados aos pacientes/participantes das pesquisas durante e após o estudo? f) Como os pacientes/participantes avaliam a experiência de participar de uma pesquisa clínica? g) Reflexões espontâneas.

Em seguida, foram feitos os recortes das unidades de registro e o ulterior agrupamento, classificação, categorização e análise destas unidades de significação. Foram consideradas como unidades de registro as ideias que respondessem às questões/categorias de análise.

Na terceira e última fase da análise de conteúdo, o tratamento dos resultados e interpretações permitiram que os conteúdos recolhidos se constituíssem em dados qualitativos e/ou análises reflexivas. Dessa forma, foram realizadas sínteses, inferências e interpretações, com articulações entre as informações obtidas e o aporte teórico da pesquisa.

A pesquisa foi aprovada pelo Sistema CEP-Conep. O projeto foi aprovado pelo Comitê de Ética em Pesquisa da Universidade Federal do Rio Grande do Norte (UFRN), conforme as normas brasileiras, mediante o parecer CAAE n. 01109412.9.0000.5292 e parecer consubstanciado n. 6137. A pesquisa, resultado de pós-doutorado no Programa de Pós-Graduação em Bioética da Universidade de Brasília, recebeu apoio financeiro do Conselho Nacional de Desenvolvimento Científico e Tecnológico (CNPq), Edital Universal, e da PróReitoria de Pesquisa da UFRN. Não há conflitos de interesses envolvidos.

\section{Perfil e aspectos envolvidos nas decisões dos participantes de ensaios clínicos}

Para melhor apresentação dos resultados, os dados quantitativos e qualitativos serão expostos conjuntamente, quando possível, de forma complementar, por categorias de análise do estudo. 
Tais resultados serão apresentados em relação aos 62 pacientes/participantes das pesquisas do centro de pesquisa e com base nas 11 entrevistas realizadas. A quase totalidade desses pacientes participou de estudo clínico fase 3; apenas 3,23\% dos pacientes estavam em estudos fase 2 e nenhum paciente participava de estudo nas fases 1 e 4.

a) Quem são esses pacientes/participantes dos ensaios clínicos?

Dos 62 pacientes, $77,42 \%$ eram do sexo feminino e $22,58 \%$ do sexo masculino, com idades distribuídas conforme o Gráfico 1.

\section{Gráfico 1}

Distribuição das idades dos pacientes participantes dos ensaios clínicos no centro de pesquisa.

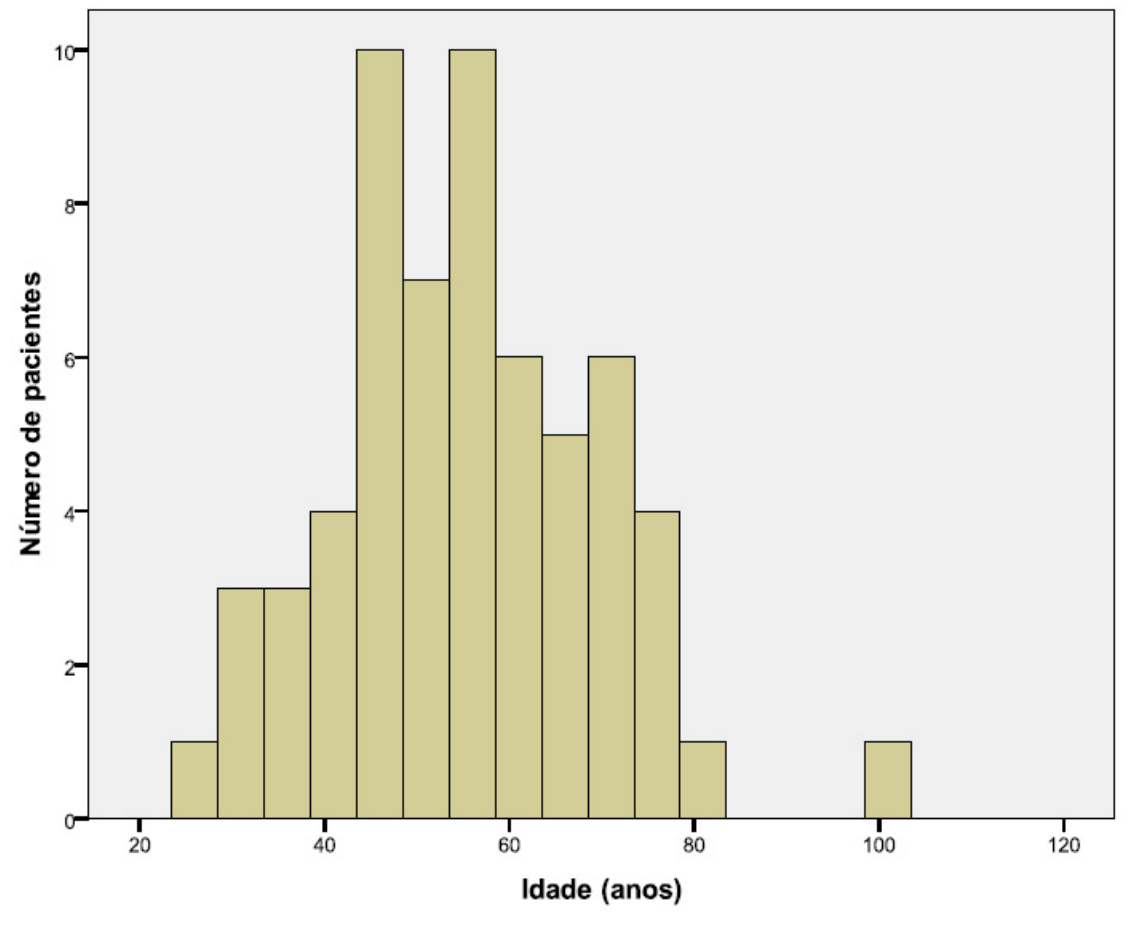

Fonte: Dados primários.

Dos 16 ensaios clínicos desenvolvidos no centro de pesquisa, 5 pesquisas $(31,25 \%)$ estavam relacionadas a cânceres exclusivos do sexo feminino (mama e ovário), das quais participaram 16 mulheres (25,80\% dos sujeitos), e apenas 2 estudos $(12,5 \%)$ eram ligados a um tumor restrito ao sexo masculino (próstata); nesses, 4 homens (6,45\% dos sujeitos) fizeram parte. Tal fator pode explicar a maior predominância da participação feminina neste caso.

A informação sobre o grau de escolaridade foi uma das questões menos respondidas nos formulários. Em mais da metade (54,84\%) essa informação 
não estava preenchida. Entre os pacientes com a escolaridade identificada, a maioria $(27,42 \%)$ tinha apenas o ensino fundamental; $11,29 \%$ eram analfabetos; $3,23 \%$ haviam concluído o ensino médio, mesma porcentagem de pacientes que possuíam ensino superior. No grupo dos 11 entrevistados, 3 não estudaram, 2 possuíam o Ensino Fundamental I incompleto e 1 o Ensino Fundamental I completo; 1 tinha o Ensino Fundamental II incompleto e 3 completaram o Ensino Fundamental II; e apenas 1 cursou o Ensino Superior.

Segundo a Pesquisa Nacional por Amostra de Domicílios realizada em 2012 pelo Instituto Brasileiro de Geografia e Estatística (IBGE), a Região Nordeste do país, região onde se localiza o centro de pesquisa estudado, é a que ainda concentra o maior número de analfabetos: $54,14 \%$ do total nacional (Brasil, 2013). Essa situação se mostra mais grave quando se consideram os analfabetos funcionais. Vale salientar que o a analfabetismo é maior entre as pessoas de mais idade, grupo que compõe a maioria dos pacientes do presente estudo.

Sobre a profissão do paciente, verificou-se que $46,78 \%$ dos participantes eram aposentados ou do lar, conforme mostra a Tabela 1.

\begin{tabular}{|c|c|c|}
\hline \multicolumn{3}{|c|}{ Distribuição por profissão dos pacientes participantes de ensaios clínicos } \\
\hline Profissão & $\mathbf{F}$ & $\%$ \\
\hline Do lar & 16 & 25,81 \\
\hline Aposentado & 13 & 20,97 \\
\hline Agricultor & 6 & 9,68 \\
\hline Comerciante & 2 & 3,23 \\
\hline Costureira & 2 & 3,23 \\
\hline Doméstica & 2 & 3,23 \\
\hline Funcionário público & 2 & 3,23 \\
\hline Professor & 2 & 3,23 \\
\hline Vendedor autônomo & 2 & 3,23 \\
\hline Auxiliar de serviços gerais & 1 & 1,61 \\
\hline Pedreiro & 1 & 1,61 \\
\hline Pensionista & 1 & 1,61 \\
\hline Sem informação & 12 & 19,35 \\
\hline Total & 62 & 100,00 \\
\hline
\end{tabular}

Fonte: Dados primários.

Pelo tipo de profissão dos sujeitos, pode-se estimar a renda familiar - quesito menos preenchido nos formulários pesquisados. Dentre os 62 pacientes, a informação sobre a renda estava disponível em apenas 13 casos. Desses, apenas 2 pacientes recebiam mais de 3 salários mínimos (SMs); os demais recebiam um valor inferior ou não apresentavam renda fixa. Em relação às pessoas entrevistadas, foi feito o seguinte registro: 6 declararam renda familiar de 1 
SM; 4 pessoas tinham renda de 2 SMs; e 1 pessoa referiu renda de 8 SMs. Os valores mencionados são referentes ao valor do SM nos anos de 2012 e 2013.

Quanto ao local de residência, em todos os formulários constava esta informação: $38,71 \%$ moravam em Natal e $61,29 \%$ em outros municípios do estado. Entre os pacientes que moravam em Natal, a maior concentração estava na Zona Norte da cidade $(14,52 \%)$ e na Zona Oeste $(12,90 \%)$, as duas regiões que apresentam a menor renda per capita, sendo a Zona Oeste a mais pobre da cidade. Não havia registros de pacientes residentes fora do estado.

Nada menos que $83,87 \%$ dos pacientes não tinham assistência privada à saúde; apenas 16,13\% contavam com essa assistência. Dos 11 entrevistados, apenas 1 declarou possuir plano de saúde privado. A gravidade da doença e a confiança depositada no centro de pesquisa estudado, por ser uma instituição de referência na área, fazem com que pessoas de todos os níveis socioeconômicos aceitem participar mais das pesquisas na área da oncologia, em comparação com outros estudos; normalmente, verifica-se nos ensaios clínicos de outras áreas a predominância de pacientes com menor grau de instrução e renda (Goldacre, 2013).

b) Como ficaram sabendo da pesquisa e por que decidiram participar?

Quando indagados a respeito da razão de terem aceitado o convite para participar de uma pesquisa clínica, verificou-se que, em síntese, as respostas se pautavam no desejo pela cura do câncer. Ou seja, nesse caso pesaram a gravidade da doença e o fato de as terapias usuais não estarem surtindo efeito. Assim, as pesquisas clínicas passaram a ser vislumbradas pelos sujeitos como a única alternativa de que dispunham. Nesse sentido, as falas que seguem são emblemáticas:

Porque tive que participar do problema da doença, né? Tava convocando, eu ia fazer o quê, né? O problema da doença eu tenho que... Não tem pra onde correr. É da doença, eu vou dizer que não vinha e quem tá doente quer ficar bom e tem que procurar um meio pra ver se realmente fica bom, se Deus quiser, né? Não tem pra onde correr $(\mathrm{S} 1)$.

Porque... sei não. (...) é porque, por exemplo, como era uma pesquisa que estava chegando, era uma droga nova, e poderia trazer a cura total da doença e a gente se conscientizou que é... Tudo que vem em prol do benefício é bom a gente fazer a tentativa, mesmo que não tenha cem por cento (S8).

Motivo com certeza de ficar boa, que eu tinha muita fé de ficar boa, que eu queria muito no momento; eu me aperreei, chorei, que dá um desespero na gente (S10).

Os pacientes também estabeleceram como razão para participar dos estudos a garantia de realização de exames e de acompanhamento médico regular 
e frequente, os quais não são garantidos de forma eficaz pelo serviço público; cabe lembrar que a renda da maioria deles torna difícil o custeio de todos os gastos com a assistência e tratamento do câncer e que apenas 1 dos entrevistados possuía plano de saúde privado. Essa necessidade esteve bem presente nas falas, como nos discursos que seguem:

A razão maior era devido aos exames serem constantes, do acompanhamento muito bom. Aí eu apostei nisso (S2).

É pelo fato de eu ser atendida mais rápido, porque tudo é mais rápido, pra fazer exame, é... Se eu tenho exame pra fazer aqui, elas mesmas tomam de conta, não precisa eu vir aqui pra marcar nada; se é consulta, do mesmo jeito, elas mesmas que resolvem tudo, só fazem me ligar e eu venho, e pronto (S10).

A procura por opções de tratamento médico pode sinalizar a precariedade de serviços de saúde disponíveis para a população, fazendo com que esses participantes busquem a alternativa das pesquisas clínicas (Oduro et al., 2008). Esse fator, apesar de mais presente nos países em desenvolvimento (Dainesi e Goldbaum, 2014; Nappo, Iafrate e Sanchez, 2013; Zammar et al., 2010; Shah et al., 2010), é encontrado em estudos em todo o mundo (Dainesi e Goldbaum, 2014; Stunkel e Grady, 2011; Kass, Maman e Atkinson, 2005).

Apenas 1 participante apontou o fato de querer contribuir com a ciência, apesar de também externar que visava ao seu próprio bem. Esse dado contraria os achados de Zammar e colaboradores (2010), nos quais o altruísmo revelouse como a principal motivação para que os pacientes brasileiros participem de ensaios clínicos, conforme visto na introdução.

O desejo de contribuir com a ciência, verbalizado por esse único participante, poderia ser interpretado, a priori, como um sentimento altruísta. Entretanto, sendo assim, parece mais adequado considerá-lo um 'altruísmo fraco ', termo que, embora originalmente proposto como descritivo de uma situação na qual os pacientes concordam em participar da pesquisa apenas porque não percebem 'nenhuma diferença líquida positiva entre os tratamentos e, portanto, não esperam perder nada, pode ser estendido para situações em que os participantes só têm interesse em ajudar os outros quando isso significar a possibilidade de ajudar a si mesmos, conforme defendem Canvin e Jacoby (2006). Em perspectiva similar, tal comportamento, também, poderia ser chamado de 'altruísmo condicional (McCann, Campbell e Entwistle, 2010).

Todos os 11 entrevistados relataram ter tomado conhecimento da pesquisa no próprio centro, quando da consulta de acompanhamento pela equipe de saúde (usualmente por um médico ou enfermeiro), sendo representativa a fala que segue: 
Eu fiquei sabendo através de doutora $X$, se eu queria fazer esse tratamento. Perguntou sobre os requisitos, eu li tudo, aí eu vi que era viável. Aí... (S2).

$\mathrm{O}$ fato de alguns pacientes, como $\mathrm{S} 2$, se referirem à pesquisa como um tratamento evidencia que eles não tiveram a compreensão, no momento em que aceitaram participar, do que significava verdadeiramente participar de um estudo clínico. Esse fato, somado à necessidade da garantia de uma boa assistência, no caso de diagnóstico de uma doença grave, suscita questionamentos a respeito da verdadeira autonomia e a exigência de uma visão mais crítica com relação ao consentimento obtido de pacientes em situação de vulnerabilidade.

Acredita-se, ainda, que a sobreposição da imagem do médico à figura do pesquisador pode fazer com que os pacientes não pensem em apresentar questionamentos (Karim et al., 1998), situação motivada pela relação de confiança e reiterada pela autoridade que esse profissional exerce (Almeida et al., 2010; Kaewpoonsri et al., 2006), aspectos evidenciados nos discursos a seguir:

Aí eu decidi porque ela (a médica) me aconselhou, disse que era uma boa opção, e então eu aceitei. Vi aquele papel que entregaram lá e vi as condições, e vou aceitar pra ver o que é que vai dar (S3).

Mas quando a médica falou, a enfermeira chamou a gente pra salinha ali, que tem do lado, que tem essa medicação, que ia dar tudo certo, aí a gente enfrentamos (S5).

Nesse cenário, é oportuno refletir sobre o duplo papel do médico, simultaneamente clínico e pesquisador, bem como sobre a confusão no entendimento do que seria pesquisa e do que seria tratamento (Timmermans e McKay, 2009). Situações desse tipo podem ser interpretadas como moralmente problemáticas, quando há exposição dos sujeitos a riscos significativos sem uma justificativa ética defensável. A confusão entre tratamento comprovado e droga experimental, cuja diferença deveria ser compreendida pelos pacientes de modo explícito por meio do TCLE, pode induzi-los a uma participação inapropriada (Gonorazky, 2011). A diferença entre tratamento comprovado e medicamento experimental deveria, inclusive, ser bem explicada no contexto da continuidade do tratamento após o fim da pesquisa, como é exigido no Brasil.

Beauchamp e Childress (2013) advertem que o termo 'pesquisa terapêutica' é potencialmente enganoso, porque, quando mal interpretado, contribui para a confusão do sujeito, pois desvia a atenção do fato de a pesquisa ainda estar em andamento. No presente estudo, por exemplo, de modo semelhante ao observado na pesquisa de Meneguin e colaboradores (2010), os resultados demonstraram que os participantes, de forma geral, desconheciam o objetivo 
da pesquisa clínica, os procedimentos metodológicos e seus efeitos adversos. Associavam os objetivos da pesquisa ao seu tratamento, acreditando, por vezes, que o protocolo de pesquisa fora desenhado com base em suas próprias necessidades e interesses.

O equívoco terapêutico é um indicador de falha relacionada ao consentimento, no qual se instala um processo baseado em uma tomada de decisão inconsciente, que compromete a avaliação dos riscos e dos benefícios por parte dos pacientes, alimentando falsas esperanças fundamentadas em decisões baseadas em critérios incorretos.

É oportuno mencionar que se tem evidenciado o conflito de interesses e os possíveis efeitos adversos decorrentes da crescente ligação dos pesquisadores, universidades e serviços de saúde com a indústria farmacêutica (Zoboli e Oselka, 2007). Todas essas questões devem ser explicitadas para os pacientes, para que eles possam compreender os possíveis conflitos de interesses que existem no cenário da pesquisa clínica.

c) Como se deu o processo de obtenção do TCLE?

Quando se trata do consentimento livre e esclarecido, a questão da escolaridade, aspecto pouco evidenciado no debate sobre pesquisa clínica envolvendo novos medicamentos, torna-se imprescindível, pois esse tipo de estudo é o que, de modo geral, oferece mais risco aos sujeitos. Dessa forma, seus participantes deveriam ter maior grau de consciência, discernimento e esclarecimento ao aceitarem participar de estudos dessa natureza.

A prevalência de participantes de menor escolaridade e de baixo nível socioeconômico está associada à menor capacidade de formular perguntas e à pouca confiança para fazê-las no momento da assinatura do TCLE (Rajaraman et al., 2011), aspectos importantes para que se possa obter uma verdadeira participação autônoma. Por isso, é necessário compreender o consentimento como um processo, e não somente como o ato de assinar um papel.

$\mathrm{Na}$ realidade, esse fator se torna ainda mais preocupante quando se analisam os TCLEs aplicados nos ensaios clínicos de medicamentos. Tais documentos são longos, apresentando, por vezes, termos complexos e linguagem médica e técnica, que mesmo aqueles com nível educacional elevado podem ter dificuldade em compreender (Passamai et al., 2012).

Levando-se em consideração o presente estudo, pode-se afirmar que a assinatura do TCLE em si é prática assegurada, pois todos os entrevistados relataram havê-la realizado no início da pesquisa, bem como assinado renovações do termo por diversas vezes. Entretanto, quando indagados sobre a compreensão do texto do documento, a maioria dos entrevistados relatou ter sido necessária ajuda para entendê-lo. Embora uma minoria tenha relatado facilidade para entender o TCLE, quando algumas questões foram aprofundadas na entrevista percebeu-se, claramente, que tinham facilidade para relatar o motivo que os levou a participar da pesquisa, mas não o entendimento da 
pesquisa em si. Assim, as falas que seguem são reveladoras da fragilidade constatada com relação ao processo da tomada de decisão:

É...mais ou menos, né? Mais ou menos. Não. Porque eu ler, eu não sou muito bem pra ler. Aí minha nora foi quem ajudou eu a ler uma parte, mais ou menos, ela foi quem leu (S1).

Umas coisas eu compreendi, né? (S3).

Ela mandou a gente levar pra casa pra ler, então eu mandei uma menina que trabalha comigo, uma secretária da escola, uma menina que trabalha na secretaria, e depois pedi pra vice-diretora ler pra mim, aí ela leu e me explicou bem direitinho (S4).

Uma parte deu muito bem pra gente entender e se conscientizar, e outras a gente ficou pensando que era uma melhora (S6).

Foi bem explicado, tudo que foi dado... Toda a equipe explicou muito bem nos deixando muito à vontade. Agora era assim, os nomes científicos, né, que a gente tinha dificuldade de entender, aí a gente perguntava (S8).

O relato dos pacientes/participantes torna perceptível que as limitações do TCLE são outro ponto que merece destaque na presente análise e em todas as reflexões, discussões e práticas que envolvam a ética em pesquisa com seres humanos. Isso é relevante, principalmente, pelo fato de a maioria delas, a exemplo das discussões provocadas por Beauchamp e Childress (2013), centrarem suas observações e críticas essencialmente no debate sobre o imprescindível respeito à autonomia decorrente de um consentimento efetivo. Observa-se, entretanto, que na prática os consentimentos têm ficado no âmbito da informação e assinatura, distantes, muitas vezes, de significarem decisões esclarecidas e conscientes. Pode-se considerar uma verdadeira indústria de consentimentos informados já incorporada de forma horizontal e acrítica, como se todas as pessoas - independentemente de nível socioeconômico e escolaridade - fossem plenamente autônomas (Garrafa, 2005).

A impressão que se tem é que as discussões na área da ética em pesquisa permeiam mais o âmbito dos microproblemas, centrado na relação profissional-paciente ou investigador-sujeito de pesquisa, não se estendendo o olhar para questões mais amplas, que envolvam a justiça social, imprescindível em cenários de desigualdades sociais. Tornar transparentes os motivos e as percepções dos pacientes pesquisados é de extrema importância e merece especial atenção diante da atual lógica de mercado que permeia a realização das pesquisas clínicas, principalmente nos cenários de desigualdades sociais. 
Em contextos nos quais as pessoas apresentam dificuldade de acesso a serviços básicos de saúde e a medicamentos, aliada a um baixo nível educacional, sem conhecimento preciso do que são as pesquisas clínicas e sobre a diferença entre estas e cuidados médicos (concebendo-os, muitas vezes, como sinônimos), há real e enorme vulnerabilidade econômica e social. Tais realidades podem se configurar como cenários propícios à suspeita repetição de pesquisas com padrões éticos diferenciados. Ao deixar de observar e discutir esse aspecto, a ciência contribui, de certa forma, para sua prática.

A realidade atual mostra que os poucos estudos que visam a analisar o perfil dos participantes dos ensaios clínicos de forma geral registram que apenas as pessoas de menor poder aquisitivo, sem acesso aos cuidados de saúde, se voluntariam para participar dessas pesquisas clínicas (Goldacre, 2013). Um estudo com o intuito de analisar estratégias para aperfeiçoar o recrutamento para ensaios clínicos realizado entre pacientes latino-americanos observou que no grupo com cuidados de saúde mais deficientes e com salários mais baixos, $96 \%$ aceitaram participar - taxa muito mais elevada do que o esperado e verificado normalmente (Sheppard et al., 2005).

Relatos provenientes de vários países indicam que a maioria dos participantes dos ensaios clínicos não entende o significado de placebo, não compreendendo que, em um ensaio controlado por placebo, há 50\% de possibilidade de o indivíduo não estar recebendo medicação (Ugalde e Homedes, 2011; Yuval et al., 2000). No Brasil, pela regra geral vigente do Sistema CEP-Conep, estudos com placebo puro não são permitidos, salvo em algumas exceções bem específicas, a exemplo das pesquisas nas quais não existam métodos provados de profilaxia, diagnóstico ou tratamento.

Pode-se afirmar que, neste trabalho, o TCLE se mostrou insuficiente para expressar uma decisão autônoma, aspecto já evidenciado em outros estudos e países (Chaisson et al., 2011; Joffe et al., 2001; Yuval et al., 2000), porém discordante dos achados de Lacativa e colaboradores (2008), em cuja pesquisa os sujeitos demonstraram compreender o conteúdo do documento que assinaram.

Conforme relatado anteriormente, todos os pacientes do presente estudo estavam vivenciando um quadro clínico grave, e os relatos dessas pessoas deram a clara impressão que o consentimento foi dado em situação de pressão e maior vulnerabilidade. Se assinassem, teriam garantida a assistência que vinham recebendo no centro de pesquisa e que todos elogiaram; caso contrário, teriam que voltar a ser atendidos pelo Sistema Único de Saúde (SUS), sem garantia de continuidade, conforme é relatado a seguir:

De uma parte é melhor porque os exames são mais rápidos, assim uma tomografia, uma cintilografia óssea. Aí facilita mais. E a gente pelo SUS marca na secretaria e passa três meses, quatro meses. A melhora que a gente acha é só isso, sabe? Entendeu? (S3). 
Mediante o que foi exposto, pode-se deduzir que, pela falta de garantia de uma assistência pública eficaz, o paciente vê a pesquisa clínica como sua única opção, sentindo-se pressionado a participar e a permanecer no estudo (Mystakidou et al., 2009). Assim, mesmo que os pacientes estejam informados de que poderão se retirar da pesquisa a qualquer momento, esse direito muitas vezes permanece apenas no nível teórico, o que compromete a sua validade moral.

d) Os pacientes/participantes conhecem os riscos e benefícios a que estiveram expostos?

Conhecer e esclarecer os riscos e os benefícios a que os participantes estão sendo ou serão expostos em uma pesquisa clínica é uma premissa ética básica. No entanto, frequentemente voluntários de ensaios clínicos assinam o documento do consentimento com pouco conhecimento sobre as informações do estudo (Minnies et al., 2008; Mandava et al., 2012). Lobato-Victoria e Pichardo-Garcia (2013) constataram que em países como o México os sujeitos de pesquisa apresentaram extrema vulnerabilidade e dependência do médico, refletidas e agravadas pelo desconhecimento das implicações relacionadas à sua participação no protocolo de pesquisa.

Muitos dos entrevistados deste estudo afirmaram não conhecer os riscos. Alguns atestaram o oposto, porém, ao serem questionados, afirmaram não se recordar de quais seriam esses riscos. Enfim, nenhum falou com convicção a respeito dos riscos, e, com relação aos benefícios, sempre os associavam ao acesso facilitado a exames e tratamento de qualidade, como nestas falas:

Riscos, eu não lembro, porque faz muito tempo que eu li, já faz uns seis meses, mas eu sei os benefícios, eles eram a assistência, que você tinha tudo programado, vinha correto, não tinha falhas podia chegar que era bem atendido; o benefício era esse e o resultado, né? Riscos até agora não, está até melhor as coisas, se eu vi risco tá embutido (S2).

Bom, ela disse que sobre os exames, tinha toda prioridade daqui e se fosse fazer algum fora, tem uns que também a pesquisa paga. A gente dá o direito de fazer, foi isso que ela explicou e foi assim que tava dando certo, estava tudo dando certo mesmo. Riscos? Não, se ela falou no momento eu não estou lembrada... (S4).

Riscos, não... não, tinha não (S7).

Foi... foi explicado tudo: os riscos e os benefícios que podia causar. Ela disse que poderia cair o cabelo, mas pra mim não tem importância, o que tem importância é a cura (S11). 
A última fala revela que, no caso do câncer, devido aos efeitos colaterais das terapias usuais, quando percebeu os riscos o paciente encarou-os como similares aos de um tratamento usual. Fica evidente também que, nesse caso, o mais importante para o paciente é a cura e não os possíveis riscos decorrentes da participação do estudo, o que corrobora outras observações registradas na literatura (Dainesi e Goldbaum, 2014). Portanto, pode-se concluir que o desconhecimento dos pacientes ou o fato de não darem importância aos riscos a que são expostos reforçam a impressão da baixa eficiência do TCLE nesta pesquisa.

e) Quais os cuidados dispensados aos sujeitos de pesquisa durante e após o estudo?

Sobre esta questão especificamente, nenhum dos participantes soube discorrer sobre os cuidados pós-estudo. A maioria afirmou, inclusive, que nada lhes foi dito a respeito; os poucos que declararam que algo foi dito não se lembravam do que havia sido exposto.

A garantia de cuidados pós-estudo não tem, aparentemente, sido rotina nos estudos do centro pesquisado, ou então esse tema não é abordado com clareza, o que suscita uma grave questão ética envolvida na discussão sobre ensaios clínicos. Uma revisão sistemática realizada a partir de ensaios clínicos sobre HIV/Aids, malária e tuberculose com o objetivo de determinar o uso de normas de cuidados e obrigações pós-estudo mostrou que a maioria dos ensaios não fez menção a esses aspectos. Dos 312 estudos, apenas 4 mencionam algo nesse sentido (Cohen et al., 2009). Grady (2005) alerta que as questões envolvendo a responsabilidade do investigador ou do patrocinador da pesquisa em garantir cuidados pós-estudo podem ser os maiores desafios éticos para os pesquisadores clínicos, especialmente aqueles envolvidos na pesquisa internacional nos próximos anos.

Na avaliação de Garrafa (2014), as modificações operadas em 2008 e 2012 na Declaração de Helsinque da Associação Médica Mundial - considerada o principal documento normativo internacional para a ética em pesquisa -, sobretudo a alteração dos seus antigos tópicos 19, 29 e 30, dirigidos à regulação ética do uso do placebo e ao acesso a benefícios ao fim do estudo, reduzem a proteção de sujeitos e comunidades socialmente vulneráveis nos países periféricos, maximizando interesses das grandes empresas farmacêuticas internacionais, apesar das crescentes críticas que suscitaram.

À época dos fatos, visando a garantir a proteção dos participantes das pesquisas no Brasil, o plenário do Conselho Nacional de Saúde, em sua centésima octogésima oitava reunião, homologou a resolução CNS 404, de 1 de agosto de 2008, da qual foram retiradas as notas de esclarecimento dos itens relacionados com cuidados de saúde a serem disponibilizados aos voluntários e ao uso de 
placebo, propostos na versão 2008 da Declaração de Helsinque. Avaliou-se que tais notas restringiam os direitos dos voluntários à assistência à saúde e, assim, foram mantidos os textos da versão 2000 do referido documento.

f) Como os pacientes/participantes avaliam a experiência de participar de uma pesquisa clínica?

A resposta a essa questão já pôde ser observada no decorrer dos discursos aqui apresentados. De forma geral, os pacientes avaliaram como muito boa ou excelente, porque eram muito bem atendidos, não precisavam esperar pelos trâmites do SUS e havia agilidade na realização de exames complementares. Entretanto, os pacientes que apresentaram efeitos colaterais indesejados, como náuseas, ou não obtiveram resposta clínica satisfatória, classificaram sua experiência como não exitosa, não avaliando tão bem a situação. Tais avaliações podem ser exemplificadas pelos depoimentos a seguir:

Eu avalio média, né? Porque eu nem participei, saí porque descontrolou meu PSA. Começou a subir e tal, tal, tal. Então eu sentia agoniado eu deixei, vim, cancelei e voltei o tratamento com dra. X. Aí fui tomar a terapia, e pronto (S6).

Eu gostei muito, eu gostei muito. Daqui eu não tenho o que dizer. É bom demais, viu? (S9).

Ave Maria! É dez! O máximo! Eu gostei muito. Fui muito bem atendida, graças a Deus, o médico muito bom, são muito bons, não tenho o que dizer não (S10).

g) Reflexões espontâneas

Nesta última categoria, serão expressas as falas espontâneas dos entrevistados, ao fim da entrevista, suscitadas pelo questionamento a respeito do desejo de se pronunciarem voluntariamente. Apenas 1 paciente não quis expressar mais nada. Os pacientes relataram sua vivência referente ao contexto de enfrentamento do câncer: alguns optaram por conversar sobre sua doença; outros agradeceram pela oportunidade de participar das pesquisas, explicitando questões como a esperança de cura ou a possibilidade de desenvolvimento de drogas eficazes, entre outras. Esse sentimento de esperança na cura e na ciência demonstrado pelos pacientes é fato, sendo oportuno ressaltar a necessidade de maior investimento público em pesquisas e do reconhecimento da saúde como direito humano fundamental, que se converte em direito social e se projeta em políticas públicas de Estado para o melhor enfrentamento das iniquidades envolvidas na questão. A propósito, vale destacar que todos os estudos realizados no centro, cenário da presente pesquisa, eram patrocinados por indústrias/laboratórios farmacêuticos. Entre os pacientes investigados, quase todos participavam de estudos propostos por instituições brasileiras 
$(45,16 \%)$ ou norte-americanas $(50 \%)$; também aparecem como proponentes instituições da Alemanha $(1,61 \%)$ e da Argentina (3,23\%).

\section{Considerações finais}

A ciência, ao longo dos tempos, proporcionou um acúmulo de conhecimentos que, por vezes, não necessariamente se pautaram por um progresso ético/ moral. A preocupação de chegar à cura das doenças e proporcionar o avanço da ciência não se projetou de forma plena sobre os sujeitos que participavam dos estudos. A verdade é que pouca atenção é dispensada a eles, mesmo com o aumento, nas últimas décadas, do número de pesquisas clínicas realizadas no Brasil, na América Latina e no mundo.

Assim, com base em um estudo de caso, buscou-se discutir uma realidade permeada por iniquidades, dar visibilidade às pessoas que participam dos ensaios clínicos e divulgar suas falas, para que possam ser ouvidas pela comunidade científica e acadêmica. Tornar transparente o olhar dos pacientes pesquisados é de extrema importância e merece especial atenção diante da atual lógica de mercado que permeia a realização das pesquisas clínicas, principalmente, nos cenários de desigualdades sociais. Mediante o presente estudo foi possível, mesmo que de forma cautelosa, evidenciar indicadores da presença ou da ausência de ações e atitudes, na prática das pesquisas clínicas, que articulam o arcabouço teórico formal a respeito da ética em pesquisa e da proteção dos sujeitos com a prática dessas pesquisas.

As decisões dos pacientes a respeito de participar de pesquisas na área da oncologia se pautaram, principalmente, na esperança de obter a cura ou melhora da sua doença, bem como na garantia de acesso a consultas, exames e tratamento de forma regular.

Evidencia-se que a assinatura do TCLE não garante a plena expressão da autonomia para todos os participantes de uma pesquisa clínica, pois as informações essenciais para uma decisão autônoma - como a devida compreensão dos objetivos do ensaio clínico, dos riscos e do direito a cuidados pós-estudo - são praticamente desconhecidas por esses participantes.

Do mesmo modo, o presente estudo evidencia que os sujeitos de pesquisa tendem a não perceber os efeitos da investigação, ou superestimam os benefícios médicos diretos de sua participação nos estudos, não apresentando uma consciência real dos riscos envolvidos e do que significa uma pesquisa clínica.

As limitações do presente estudo, principalmente pelo fato de haver sido realizado em apenas um centro de pesquisa localizado no Nordeste do país e com um número reduzido de participantes, não permitem que represente o cenário global da pesquisa clínica no Brasil. Mas a intenção maior, neste momento, não é generalizar a situação revelada, mas contribuir com as reflexões, 
discussões e práticas em pesquisa clínica, além de estimular estudos similares nesse segmento, visando a um melhor conhecimento dessa complexa questão.

Espera-se, portanto, que os resultados ora apresentados possam incitar ao exercício e ao diálogo mais críticos entre os diferentes atores e instituições envolvidos na área da pesquisa com seres humanos, mormente nas pesquisas clínicas. Assim, busca-se promover uma ciência consciente e responsável, capaz de impedir que pessoas, principalmente de países pobres e sem acesso aos cuidados de saúde, sejam submetidas a situações de desigualdade, vulnerabilidade e sofrimento moral.

Concentrar a atenção nos riscos a que estão expostos os pacientes socialmente vulneráveis nas pesquisas clínicas, em um contexto de saúde cada vez mais neoliberal, pode propiciar uma visão mais crítica sobre as questões complexas e os desafios que permeiam o terreno dos ensaios clínicos globais terceirizados, principalmente em países pobres e em desenvolvimento. É essencial ampliar o compromisso com o social de maneira concreta e crítica, de forma a diminuir as disparidades, adaptar-se às realidades de cada contexto e extinguir de uma vez por todas a exploração que vitimiza incontáveis sujeitos de pesquisa por todo o mundo.

\section{Colaboradores}

Karla Patrícia Cardoso Amorim é responsável pela concepção da pesquisa, participando de todas as etapas desde o planejamento, desenvolvimento e elaboração e revisão do artigo. Volnei Garrafa participou da concepção do estudo, análise de dados e na elaboração e revisão do artigo. Alana Dantas de Melo, Andressa Vellasco Brito Costa, Gabriella Caldas Leonardo Oliveira, Heitor Giovanni Lopes, Eduardo Judene da Silva Pereira e Francisco Ademar Fernandes Júnior participaram da coleta dos dados, análise de dados redação e revisão do artigo. 
Resumen Estudio sobre los participantes de ensayos clínicos en el área de oncología, discutiendo la paradoja entre avance científico y desigualdad social. Se buscó conocer quiénes son esas personas y analizar los aspectos involucrados en sus decisiones, con base en entrevistas y documentos. Hubo mayor participación femenina. La tendencia es que los participantes tienen pocos años de estudio formal y bajos ingresos. La mayoría es jubilada y ama de casa, y no tiene asistencia médica privada. Sus decisiones giraron en torno a la búsqueda de la cura o una mejora, y el acceso regular garantizado a la atención médica integral y a los medicamentos. La firma del formulario de consentimiento voluntario e informado no constituyó una expresión de autonomía, ya que información esencial como objetivos, riesgos y cuidados posteriores al estudio es prácticamente desconocida. Los participantes de la investigación tienden a no comprender los objetivos de la investigación, o sobreestiman los beneficios médicos directos de su participación, sin ser conscientes de los riesgos implicados y lo que significa una investigación. Los resultados deberían incitar al ejercicio y al diálogo más críticos entre los diferentes actores e instituciones involucrados en el área de la investigación con seres humanos, a fin de promover un entendimiento consciente y responsable, que impida que las personas sean colocadas en situación de desigualdad, vulnerabilidad y sufrimiento moral.

Palabras clave sujetos de investigación; ensayos clínicos; desigualdad social; ética en investigación; neoplasias.

\section{Notas}

${ }^{1}$ Universidade Federal do Rio Grande do Norte, Departamento de Medicina Clínica, Natal, Rio Grande do Norte, Brasil.

<amorimkarla@yahoo.com.br>

Correspondência: Universidade Federal do Rio Grande do Norte, Centro de Ciências da Saúde, Avenida Cordeiro de Farias, s/n, Petrópolis, CEP 59012-300, Natal, Rio Grande do Norte, Brasil.

${ }^{2}$ Universidade de Brasília, Programa de Pós-Graduação em Bioética, Brasília, Distrito Federal, Brasil.

<garrafavolnei@gmail.com>

${ }^{3}$ Universidade Federal do Rio Grande do Norte, Natal, Rio Grande do Norte, Brasil. <alanadantas@gmail.com>

${ }^{4}$ Universidade Federal do Rio Grande do Norte, Natal, Rio Grande do Norte, Brasil. $<$ andressavellasco04@gmail.com>

${ }^{5}$ Universidade Federal do Rio Grande do Norte, Natal, Rio Grande do Norte, Brasil. <gabi.leonardo.187@gmail.com>

${ }^{6}$ Universidade Federal do Rio Grande do Norte, Natal, Rio Grande do Norte, Brasil. $<$ heitorgl@gmail.com>

${ }^{7}$ Universidade Federal do Rio Grande do Norte, Natal, Rio Grande do Norte, Brasil. <eduardo.med.ufrn@gmail.com>

${ }^{8}$ Universidade Federal do Rio Grande do Norte, Natal, Rio Grande do Norte, Brasil. <ademar.ufrn@gmail.com> 


\section{Referências}

ALMEIDA, Carolina H. et al. A pesquisa científica na saúde: uma análise sobre a participação de populações vulneráveis. Texto \& Contexto Enfermagem, Florianópolis, v. 19, n. 1, p. 104-111, 2010.

BARDIN, Laurence. Análise de conteúdo. Ed. rev. e atual. Lisboa: Edições 70, 2009.

BEAUCHAMP, Tom L; CHILDRESS, James F. Principles of biomedical ethics. 7. ed. New York: Oxford University, 2013.

BRASIL. Ministério do Planejamento, Orçamento e Gestão. Instituto Brasileiro de Geografia e Estatística (IBGE). Diretoria de Pesquisas Coordenação de Trabalho e Rendimento. Pesquisa Nacional por Amostra de Domicílios: síntese de indicadores 2012. Rio de Janeiro: IBGE. 2013. Disponível em: <http://biblioteca.ibge.gov. br/visualizacao/livros/liv65857.pdf $\geq$. Acesso em: 14 ago. 2015.

CANVIN, Krysia; JACOBY, Ann. Duty, desire or indifference? A qualitative study of patient decisions about recruitment to an epilepsy treatment trial. Trials, London, v. 7, n. 32, 2006. Disponível em: <http://www.trialsjournal.com/content/7/1/32 2 . Acesso em: 18 jul. 2017.

CHAISSON, Lelia H. et al. Repeated assessments of informed consent comprehension among HIV-infected participants of a threeyear clinical trial in Botswana. PLoS One, San Francisco, v. 6, n. 10, p. e22.696, 2011.

COHEN, Emma R. M. et al. Reporting of informed consent, standard of care and post-trial obligations in global randomized intervention trials: a systematic survey of registered trials. Devel World Bioethics, Quenzan City, v. 9, n. 2, p. 74-80, 2009.

DAINESI, Sonia; GOLDBAUM, Moisés. Reasons behind the participation in biomedical research: a brief review. Revista Brasileira de Epidemiologia, São Paulo, v. 17, n. 4, p. 842-851, out.-dez. 2014.
GARRAFA, Volnei. Da bioética de princípios a uma bioética interventiva. Bioética, Brasília, v. 13, n. 1, p. 125-134, 2005.

GARRAFA, Volnei. Declaración de Helsinki y sus repetidos 'ajustes': un tema fatigoso... Revista Lasallista de Investigación, Antioquia, v. 11, n. 1, p. 35-40, 2014.

GARRAFA, Volnei; LORENZO, Cláudio. Helsinque 2008: redução de proteção e maximização de interesses privados. Revista da Associação Médica Brasileira, São Paulo, v. 55, n. 5, p. 514-518, 2009.

GOLDACRE, Ben. Farmacêuticas da treta: como as empresas da indústria farmacêutica induzem os médicos em erro e fazem mal aos doentes. Lisboa: Editorial Bizâncio, 2013.

GONORAZKY, Sergio E. Principio de precaución en investigación biomédica, seguridad, obligaciones post-investigación y eficacia terapéutica supuesta de las drogas experimentales: violaciones a la dignidad de los pacientes. Salud Colectiva, Buenos Aires, v. 7, n. 2, p. 149-151, 2011.

GRADY, Christine. The Challenge of assuring continued post-trial access to beneficial treatment. Yale Journal of Health Policy, Law, and Ethics, New Haven, v. 5, n. 1, p. 425436, 2005

INSTITUTO NACIONAL DE CÂNCER JOSÉ ALENCAR GOMES DA SILVA (INCA). Estimativa 2016: incidência de câncer no Brasil. Rio de Janeiro: Inca, 2015. Disponível em: $<$ http://www.inca.gov.br/wcm/dncc/2015/ estimativa-2016.asp> . Acesso em: 17 jul. 2017.

JOFFE, Steven et al. Quality of informed consent in cancer clinical trials: a cross-sectional survey. The Lancet, London, v. 358, n. 9.295, p. 1.772-1.777, 2001.

KAEWPOONSRI, Nantawan et al. Factors related to volunteer comprehension of informed consent for a clinical trial. Southeast Asian Jour- 
nal of Tropical Medicine and Public Health, Bangkok, v. 37, n. 5, p. 996-1.004, 2006.

KARIM, Quarraisha A. et al. Informed consent for HIV testing in a South African hospital: is it truly informed and truly voluntary? American Journal of Public Health, Washington, v. 88, n. 4, p. 637-640, 1998.

KASS, Nancy E.; MAMAN, Suzanne; ATKINSON, Joan. Motivations, understanding, and voluntariness in international randomized trials. IRB: Ethics \& Human Research, New York, v. 27, n. 6, p. 1, 2005.

LACATIVA, Paulo G. S. et al. Perfil de sujeitos de pesquisa clínica em um centro ambulatorial independente. Ciência \& Saúde Coletiva, Rio de Janeiro, v. 13, n. 3, p. 1.023-1.032, 2008.

LOBATO-VICTORIA, Amanda Cecilia; PICHARDOGARCIA, Luz María G.. ¿ En qué medida es verdad que los sujetos de investigación comprenden sus derechos? Persona y Bioética, Chia, v. 17, n. 1, p. 49-57, 2013.

MANDAVA, Amulya et al. The quality of informed consent: mapping the landscape: a review of empirical data from developing and developed countries. Journal of Medical Ethics, London, v. 38, n. 6, p. 356-365, 2012.

MCCANN, Sharon K.; CAMPBELL, Marion K.; ENTWISTLE, Vikki A. Reasons for participating in randomized controlled trials: conditional altruism and considerations for self. Trials, London, v. 11, p. 11-31, 2010.

MENEGUIN, Silmara et al. Entendimento do termo de consentimento por pacientes partícipes em pesquisas com fármaco na cardiologia. Arquivos Brasileiros de Cardiologia, Rio de Janeiro, v. 94, n. 1, p. 4-9, 2010.

MINAYO, Maria Cecília S. O desafio do conhecimento: pesquisa qualitativa em saúde. 7. ed. São Paulo: Hucitec, 2000.

MINNIES, Deon et al. Evaluation of the quality of informed consent in a vaccine field trial in a developing country setting. BMC Medical Ethics, London, v. 9, n. 1, p. 15, 2008.

MYSTAKIDOU, Kyriaki et al. Ethical and practical challenges in implementing informed consent in HIV/AIDS clinical trials in developing or resource-limited countries. Sahara-J: Journal of Social Aspects of HIV/Aids, Pretoria, v. 6, n. 2, p. 46-57, 2009.

NAPPO, Solange A.; IAFRATE, Giovanna B.; SANCHEZ, Zila M. Motives for participating in a clinical research trial: a pilot study in Brazil. BMC Public Health, London, v. 13, n. 1, p. 19, 2013.

ODURO, Abraham R. et al. Understanding and retention of the informed consent process among parents in rural northern Ghana. $B M C$ Medical Ethics, London, v. 9, n. 1, p. 12, 2008.

PASSAMAI, Maria da Penha B. et al. Functional health literacy: reflections and concepts on its impact on the interaction among users, professionals and the health system. Interface: Comunicação, Saúde, Educação, Botucatu, v. 16, n. 41, p. 301-314, 2012.

RAJARAMAN, Divya et al. How participatory is parental consent in low literacy rural settings in low income countries? Lessons learned from a community based study of infants in South India. BMC Medical Ethics, London, , v. 12, n. 1, p. 3, 2011.

SHAH, Jatin Y. et al. What leads Indians to participate in clinical trials? A meta-analysis of qualitative studies. PLoS One, San Francisco, v. 5, n. 5, p. e10.730, 2010.

SHEPPARD, Vanessa B. et al. Brief report: if you build it, they will come. Journal of General Internal Medicine, Alexandria, v. 20, n. 5, p. 444-447, 2005.

STUNKEL, Leanne; GRADY, Christine. More than the money: a review of the literature examining healthy volunteer motivations. Contemporary Clinical Trials, New York, v. 32, n. 3, p. 342-352, 2011. 
TIMMERMANS, Stefan; MCKAY, Tara. Clinical trials as treatment option: bioethics and health care disparities in substance dependency. Social Science \& Medicine, New York, v. 69, n. 12, p. 1.784-1.790, 2009.

UGALDE, Antonio; HOMEDES, Núria. Cuatro palabras sobre ensayos clínicos: ciencia/negocio, riesgo/beneficio. Salud Colectiva, Buenos Aires, v. 7, n. 2, p. 135-148, 2011.

YUVAL, Rita et al. Patient comprehension and reaction to participating in a double-blind randomized clinical trial (ISIS-4) in acute myocardial infarction. Archives of Internal Medicine, Chicago, v. 160, n. 8, p. 1.142-1.146, 2000.
ZAMMAR, Guilherme et al. So different, yet so similar: meta-analysis and policy modeling of willingness to participate in clinical trials among Brazilians and Indians. PLoS One, San Francisco, v. 5, n. 12, e14.368, 2010.

ZOBOLI, Elma L. C. P.; FRACOLLI, Lislaine A. A vulnerabilidade do sujeito de pesquisa: uma abordagem multidimensional. Cadernos de Ética em Pesquisa, Brasília, v. 8, p. 20-21, 2001.

ZOBOLI, Elma; OSELKA, Gabriel. Conflito de interesses na pesquisa clínica. Bioética, Brasília, v. 15, n. 1, p. 65-76, 2007.

Recebido em 04/05/2017. Aprovado em 30/10/2017. 